\title{
INVESTIGATING THE EFFECT OF A VISUAL SEARCH TASK FOR SIMULATOR- BASED DRIVER TRAINING
}

\author{
P.M. van Leeuwen, R. Happee \& J.C.F. de Winter \\ Biomechanical Engineering, Faculty of Mechanical, Maritime and Materials Engineering, \\ Delft University of Technology \\ Delft, The Netherlands \\ Email: P.M.vanLeeuwen@TUDelft.nl
}

\begin{abstract}
Summary: Novice drivers tend to direct their gaze to the road ahead and not scan the environment properly. This study investigated the training effectiveness of a visual search task in a driving simulator, aimed at increasing young drivers' spread of visual search. Two groups of inexperienced drivers were instructed to drive as accurately as possible in the center of the right lane in a self-paced driving task of four 6-min sessions in a rural environment. While driving, one group performed a visual search task, consisting of detecting and fixating on visual stimuli in the peripheral area. The stimuli were purple dots that faded in slowly and disappeared when fixated by the participant. After training, both groups drove a transfer session in an urban environment, in which various hazardous situations occurred. Results showed that both groups improved their lane keeping performance, whereas the training group became more proficient in the visual search task. However, in the transfer session no group differences were detected. In conclusion, despite improvements in visual search performance during a relatively short training period, the visual search training did not detectibly influence the spread of visual search of novice drivers during a post training transfer session.
\end{abstract}

\section{INTRODUCTION}

Young drivers are overrepresented in road traffic crashes. Crash rates are highest in the first months of independent driving and decline as drivers gain experience (Mayhew, Simpson, \& Pak, 2003). Many studies have shown differences in visual search behavior between experienced and novice drivers (e.g., Underwood, Chapman, Brocklehurst, Underwood, \& Crundall, 2003). One factor that has been associated with the high crash rates among novice drivers is their poor ability to identify and anticipate hazards (e.g., Fisher, Pollatsek, \& Pradhan, 2006). McKnight and McKnight (2003) reviewed 2,000 police accident reports, and showed that failure to search ahead, to the side, or the rear was a factor in $43 \%$ of young drivers' crashes.

Several studies have shown that novice drivers have an elevated mental workload (Lee, 2007), a phenomenon which has been associated with spatial gaze concentration (Recarte \& Nunes, 2003). Crundall and Underwood (1998) found that inexperienced drivers are less inclined than experienced drivers to adjust their visual search to the complexity of the environment and to changing task demands. Novices tend to allocate their visual attention to information close to the vehicle, which may be caused by their limited steering control skills compared to experienced drivers (Mourant \& Rockwell, 1972). Summala, Nieminen, and Punto (1996) showed that inexperienced drivers rely less on peripheral vision for lateral vehicle control, and fixate more on lane markers and areas close to the vehicle. An effect of driving experience on peripheral vision 
was also found by Crundall, Underwood, and Chapman (1999). They reported decreased peripheral detection rates for non-drivers versus experienced drivers while watching video clips of driving scenes. Underwood, Chapman, Bowden \& Crundall (2002) showed that novices’ reduced visual search on divided highways is caused by an impoverished mental model of likely events to occur, instead of being caused by cognitive demands due to lack of driving experience.

In this study, we investigated the effectiveness of a visual search task aimed at increasing inexperienced drivers' spread of search in a driving simulator. Inexperienced drivers were instructed to perform a lane keeping task while peripheral visual cues were to be detected and fixated. Low saliency and random appearance of these stimuli prevented bottom up (i.e., stimulus driven) responses, resulting in active visual search.

\section{METHOD}

Two groups of inexperienced drivers were tested; see Table 1 for an overview. One group received a visual search training (14 participants) and a control group (16 participants) drove without visual search training. Groups were assigned balancing age, gender, driving simulator experience, total mileage, and months since obtaining the driving license, using the minimization method of Taves (1974). All participants received a compensation of 5 euro and provided written informed consent.

Table 1. Mean demographic and driving experience data (standard deviations in parentheses)

\begin{tabular}{lccc}
\hline & Control & & Training \\
$\begin{array}{l}\text { Age (years) } \\
\text { Gender (male / female) }\end{array}$ & $19.1(1.3)$ & & $19.1(0.8)$ \\
$\begin{array}{l}\text { Driving simulator } \\
\text { experience (number of } \\
\text { participants) }\end{array}$ & 2 & $13 / 1$ \\
$\begin{array}{l}\text { Driving license (months) } \\
\text { Total mileage (km) }\end{array}$ & $6.6(3.8)$ & & $6.8(3.5)$ \\
$\quad 0-10,000$ & 15 & & 14 \\
$10,000-20,000$ & 1 & & 0 \\
\hline
\end{tabular}

This study used a fixed-base simulator (Green Dino BV), with 180-degree horizontal and 45-degree vertical field of view and surround sound simulating a middle class passenger vehicle. The virtual world was projected using three LCD projectors with 1024 x 768 pixels for the center display and $800 \times 600$ pixels for the two side displays. Instruments and mirrors were integrated in the simulation visualisation.

The visual search training consisted of randomly appearing purple dots left and right of the road, above the virtual hood, and below the rear view mirror (Figure 1). The dots were $20 \mathrm{~mm}$ in diameter and were composed of the folowing RGB color components: 255, 87, 213. The dots faded in, in $7.6 \mathrm{~s}$. On average 29 (SD = 4.0) dots appeared per training session. When subjects fixated on a dot for $350 \mathrm{~ms}$ the dot would disappear and a next dot would randomly appear within 5 to 9 seconds. Non-fixated dots remained visible for 5 to 9 seconds, after which they disappeared and a new dot appeared. A two degree fixation tolerance was used to account for eye tracker inaccuracy. Gaze was recorded at $60 \mathrm{~Hz}$ using a three-camera remote mounted Smart Eye (version 5.6) eye tracker.

Participants drove four training sessions and one transfer session of six minutes, each followed by approximately 5 min breaks, in which subjects completed the NASA TLX questionnaire for measuring workload (NASA, 1986). After each training session the training group received oral 
feedback on the number of detected dots, motivating them to improve their search performance. During the transfer session the visual search task was disabled.

All training sessions took place on a two-lane rural road, with various sharp curves, and without other traffic, see Figure 1. The instructed task was to keep the vehicle as accurately as possible in the center of the right lane. Furthermore, participants were instructed to follow the Dutch traffic rules and drive within the $80 \mathrm{kph}$ speed limit. The transfer session took place in an urban environment with short rural road sections. The urban environment consisted of 30, 50 and 80 kph speed limit zones with other traffic (cars, cyclists, and pedestrians). During the transfer session various hazardous situations occurred (e.g., crossing pedestrian) triggered after passing fixed locations in the virtual world.
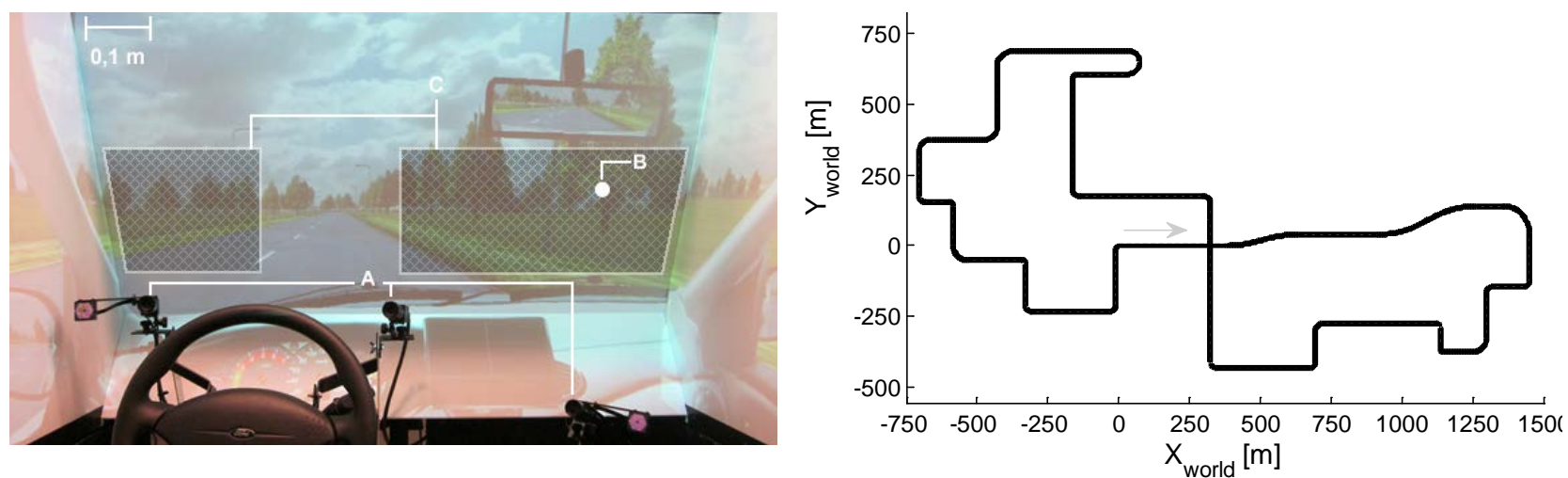

Figure 1. Left: Simulator during the experiment $(A=$ eye tracker, $B=$ example of an appearing dot, $C=$ areas where the dots appeared). Right: Top view of the course; arrow indicates starting point and driving direction

Gear selection was automated; participants were required to steer, accelerate, and brake. Participants were informed in writing of the presence of the eye tracker and, if applicable, the visual search training during the training sessions. After taking place in the simulator the eye tracker was calibrated and the instructions were repeated on the screen. After the fourth training session, the participants received on-screen information regarding the changed driving scenario in the transfer phase.

The following dependent measures were determined per session:

1. Mean speed (m/s).

2. Standard deviation lateral position (SDLP) (m).

3. Gaze road center (GRC) (\%), the percentage of time gazed within an 8 degree radius around the road center.

4. Horizontal gaze variance (HGV) $\left(\mathrm{deg}^{2}\right)$ was calculated on the straight road segments and was used as measure of spread of search.

5. Steering reversal rate, (\#/min) defined as the number of changes in steering wheel direction per minute with the steering velocity exceeding 3 degrees per second. This measure was calculated from the $3 \mathrm{~Hz}$ low pass filtered steering wheel angle.

6. Targets missed (\#).

7. Mean target response time (s). Missed targets were excluded from the analysis.

8. NASA TLX (\%), a workload assessment tool in the form of a questionnaire. 
The results were compared per session between the training and control group using a twosample $t$ test. The NASA TLX results were fractionally ranked prior to statistical analysis because of their skewed distribution. Eye tracker data from $0.5 \mathrm{~s}$ before until $0.5 \mathrm{~s}$ after sequences of lost data (e.g., due to blinks) were removed. If more than $60 \%$ of eye tracker data was removed from a session, the corresponding session was removed from the analysis.

\section{RESULTS}

One participant ended the experiment due to simulator discomfort and was replaced by another participant. Dependent measures per group and session are shown in Table 2. None of the training group participants reported targets failing to disappear after being fixated as a result of eye tracker inaccuracy or loss of tracking.

Table 2. Averaged group results and corresponding $p$ values for the control group $(n=16)$ and training group $(n=14)$ in training and transfer sessions (standard deviations in parentheses)

\begin{tabular}{|c|c|c|c|c|c|c|}
\hline & \multicolumn{4}{|c|}{ Training } & \multirow{2}{*}{$\begin{array}{c}\text { Transfer } \\
5 \\
\end{array}$} \\
\hline & & 1 & 2 & 3 & 4 & \\
\hline \multirow{3}{*}{ Mean speed (m/s) } & Control & $17.2(1.5)$ & $17.0(1.8)$ & $16.7(1.8)$ & $17.1(1.8)$ & $13.0(0.83)$ \\
\hline & Training & $17.3(1.4)$ & $17.1(1.7)$ & $17.0(1.5)$ & $17.4(1.4)$ & $12.9(0.79)$ \\
\hline & $p$ & .787 & .833 & .657 & .590 & .823 \\
\hline \multirow{3}{*}{$\begin{array}{l}\text { Standard deviation } \\
\text { lateral position (m) }\end{array}$} & Control & $0.73(0.20)$ & $0.52(0.16)$ & $0.46(0.07)$ & $0.46(0.13)$ & $0.59(0.16)$ \\
\hline & Training & $0.75(0.21)$ & $0.67(0.15)$ & $0.52(0.09)$ & $0.55(0.13)$ & $0.58(0.15)$ \\
\hline & $p$ & .725 & .021 & .105 & .146 & .858 \\
\hline \multirow{3}{*}{ Gaze road center* (\%) } & Control & $62(6.8)$ & $58(7.0)$ & $59(7.2)$ & 57 (8.3) & $39(5.0)$ \\
\hline & Training & $54(10.3)$ & $50(11.3)$ & 49 (7.3) & $46(8.2)$ & $37(7.8)$ \\
\hline & $p$ & .027 & .029 & .001 & $<.001$ & .411 \\
\hline \multirow{3}{*}{$\begin{array}{l}\text { Horizontal gaze } \\
\text { variance* }^{*}\left(\mathrm{deg}^{2}\right)\end{array}$} & Control & $51.8(27.4)$ & $76.4(38.1)$ & 86.7 (51.5) & $96.9(51.0)$ & $162.2(48.3)$ \\
\hline & Training & 90.7 (30.8) & 137.7 (47.7) & $133.1(53.2)$ & $170.0(57.6)$ & $176.5(98.2)$ \\
\hline & $p$ & .002 & .001 & .034 & .002 & .639 \\
\hline \multirow{3}{*}{$\begin{array}{l}\text { Steering reversal rate } \\
(\# / \mathrm{min})\end{array}$} & Control & 20.1 (5.3) & $15.8(3.0)$ & 14.1 (3.3) & $13.8(3.2)$ & 15.5 (3.3) \\
\hline & Training & $20.2(3.0)$ & 16.4 (3.3) & $16.6(3.5)$ & $17.3(3.3)$ & $15.1(3.6)$ \\
\hline & $p$ & .973 & .630 & .086 & .016 & .760 \\
\hline Targets missed (\#) & Training & $6.93(2.64)$ & $4.07(2.81)$ & 2.57 (1.91) & $2.14(2.07)$ & - \\
\hline \multirow[t]{2}{*}{$\begin{array}{l}\text { Mean target response } \\
\text { time (s) }\end{array}$} & Training & $5.09(0.74)$ & $3.92(0.47)$ & $3.73(0.61)$ & $3.34(0.66)$ & - \\
\hline & Control & 47 (14) & $44(15)$ & 37 (16) & 38 (17) & 49 (18) \\
\hline \multirow[t]{2}{*}{ NASA TLX (\%) } & Training & $57(16)$ & 49 (17) & 41 (17) & $41(14)$ & 49 (19) \\
\hline & $p$ & .081 & .397 & .489 & .601 & .989 \\
\hline
\end{tabular}

*on average $25 \%$ of eye tracker data was discarded per session. In total 14 sessions were removed from the analysis.

During the training sessions, a significantly higher visual search (i.e., lower GRC, higher HGV) was observed for the training group compared to the control group, illustrated by Figure 2. 

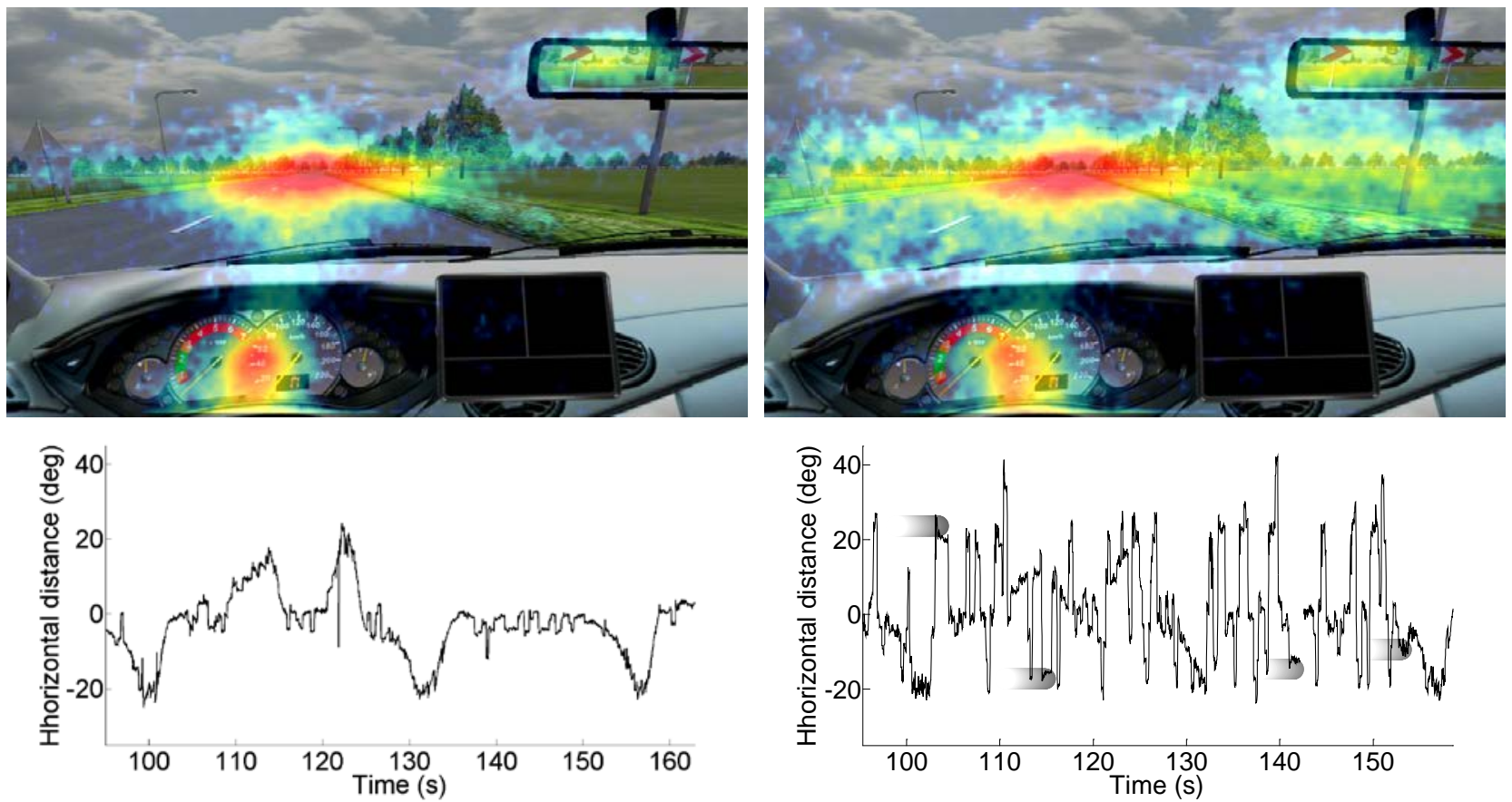

Figure 2. Gaze distribution for the control (top left) and training group (top right) for all participants in the 4th session. Distributions were generated after converting to a logarithmic scale. Horizontal gaze angle for two selected participants; control group (bottom left) and training group (bottom right). Fading in of the targets is illustrated by the increasing grayscale intensity (bottom right only)

The SDLP for the training group was significantly higher than the SDLP of the control group in the second training session only. Steering reversal rate was significantly higher for the training group in the last training session only. No significant group differences were found regarding mean speed and self reported workload. No significant differences between the training and control group were found in the transfer session for any of the driving behavior, eye-scanning, or workload measures.
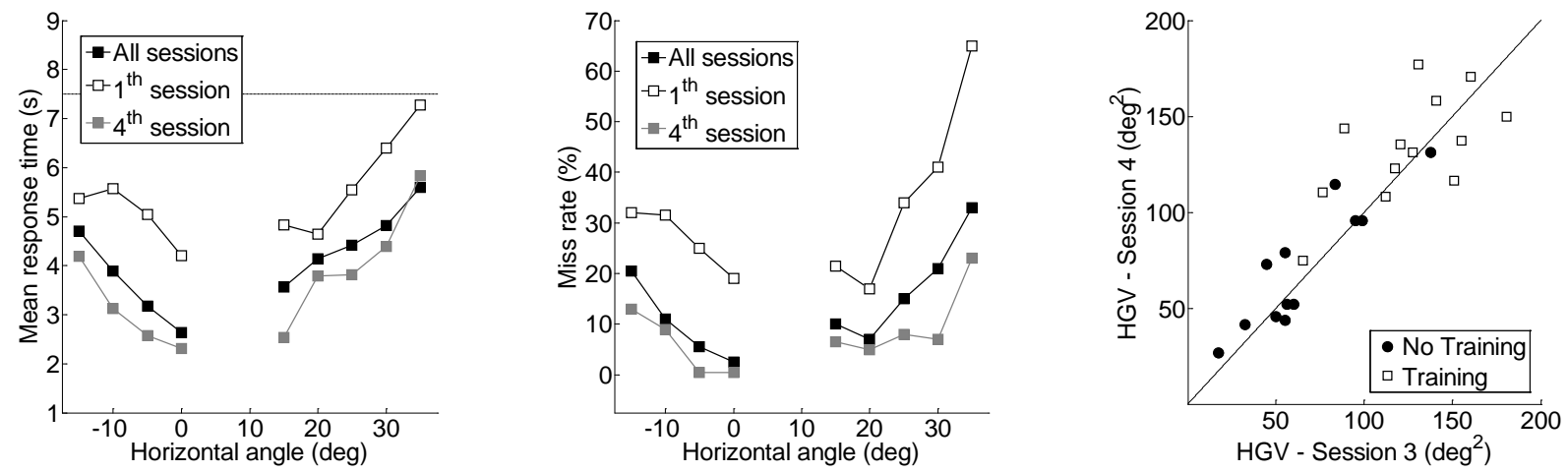

Figure 3. Left: Distribution of mean response time as a function of the horizontal view angle for session 1, session 4, and averaged across all four sessions. The dashed line indicates the time at which the target dot had completely faded in. Center: Percentage of missed targets as a function of horizontal view angle for session 1, session 4 and averaged across all four sessions. Right: Horizontal gaze variance for participants of both groups in sessions 3 and 4 . Five participants are missing from the figure due to missing eye tracker values

The HGV significantly increased from the first session to the last training session for the training group $(t(13)=4.52, p<.001)$ and for the control group $(t(14)=3.29, p=.006)$. A significant performance improvement from the first training session to the last training session was found for 
missed targets $(t(13)=7.81, p<.001)$ and target response time $(t(13)=7.20, p<.001)$. Figure 3 shows that response time and target miss rate are higher for more peripheral targets, and that gaze variance differs strongly between participants and is consistent between sessions 3 and 4 .

\section{DISCUSSION}

In this study, we investigated the training effectiveness of a visual search task. The group who performed the visual search task while driving became gradually better at detecting the visual stimuli, as demonstrated by a diminished number of object misses and significantly improved response time. In the transfer session, no differences in eye-scanning and driving behavior were detected between the two groups, indicating that the training effects did not detectibly generalize to the new condition.

The ineffectiveness of the visual search training may be explained by the absence of hazardous information in the visual stimuli. The stimuli were designed to prevent bottom up responses during driving, aimed at improving the spread of visual search. The 'meaningless' visual search however, may not have improved drivers' mental model of hazardous situations. Improving the novices' mental model of hazardous situations could result in improved recognition and processing of hazards (Chapman, Underwood, \& Roberts, 2002). Furthermore, due to the lack of hazardous information in the visual stimuli the inexperienced drivers were not trained in generating appropriate responses after detecting hazardous situations.

Driving performance and visual search showed strong performance improvement for the visual training group during training, indicating increasingly effective timesharing between both tasks. Schneider and Fisk (1982) found that two visual search tasks can be more easily timeshared when both tasks are cognitively automated, or when one task is automated and the other is a controlled search task. Possibly, the visual attention required for vehicle control in the training sessions was an automated task, which therefore could easily be timeshared with the visual search task.

The sample tested in this study consisted mainly of male students, limiting the generalizability of the results. Furthermore, the training lasted only 24 minutes per participant, whereas driving skill is developed during years of driving experience (Mayhew et al., 2003). Other training interventions aimed at improving young drivers' mental model of hazards (e.g., Fisher et al., 2006) seem effective in improving novices' visual search in hazardous situations. Training of visual search by manipulating drivers’ eye-scanning must be addressed carefully, however. Training visual search during a driving task for which trainees lack attentional resources may well decrease driving safety (Crundall et al., 2012), as redirecting their visual attention may reduce their attention to other vital visual tasks.

\section{ACKNOWLEDGMENTS}

We thank J. Damsteeg, J. van Ginkel, B. van Sambeek and T. Vlaar for their contribution in conducting the experiments. Some results have been presented at the 2012 ICTTP conference in Groningen. The research is supported by the Dutch Ministry of Economic affairs through the project Driver Observation in Car Simulators (HTASI09004-DrivObs) and by the Dutch Technology Foundation (STW), applied science division of the Netherlands Organisation for Scientific Research (NWO). 


\section{REFERENCES}

Chapman, P., Underwood, G., \& Roberts, K. (2002). Visual search patterns in trained and untrained novice drivers. Transportation Research Part F: Traffic Psychology and Behaviour, 5(2), 157-167.

Crundall, D., \& Underwood, G. (1998). Effects of experience and processing demands on visual information acquisition in drivers. Ergonomics, 41(4), 448-458.

Crundall, D., Underwood, G., \& Chapman, P. (1999). Driving experience and the functional field of view. Perception, 28(9), 1075-1088.

Crundall, D., Chapman, P., Trawley, S., Collins, L., van Loon, E., Andrews, B., \& Underwood, G. (2012). Some hazards are more attractive than others: Drivers of varying experience respond differently to different types of hazard. Accident Analysis \& Prevention, 45, 600-609.

Fisher, D. L., Pollatsek, A. P., \& Pradhan, A. (2006). Can novice drivers be trained to scan for information that will reduce their likelihood of a crash? Injury Prevention, 12, 25-29.

Lee, J. D. (2007). Technology and the teen driver. Journal of Safety Research, 38, 203-213.

Mayhew, D. R., Simpson, H. M., \& Pak, A. (2003). Changes in collision rates among novice drivers during the first months of driving. Accident Analysis \& Prevention, 35, 683-691.

McKnight, A. J., \& McKnight, A. S. (2003). Young novice drivers: careless or clueless? Accident Analysis \& Prevention, 35(6), 921-925.

Mourant, R. R., \& Rockwell, T. H. (1972). Strategies of visual search by novice and experienced drivers. Human Factors,14(4), 325-335.

NASA (1986). Task Load Index (NASA-TLX). v. 1.0. Paper and pencil package (instruction manual). Moffett Field, CA: NASA Ames Research Center. Retrieved from http://humanfactors.arc.nasa.gov/groups/TLX/downloads/TLX.pdf

Recarte, M. A., \& Nunes, L. M. (2003). Mental workload while driving: effects on visual search, discrimination, and decision making. Journal of Experimental Psychology: Applied, 9(2), 119-137.

Schneider, W., \& Fisk, A. D. (1982). Concurrent automatic and controlled visual search: Can processing occur without resource cost? Journal of Experimental Psychology: Learning, Memory, and Cognition, 8(4), 261-278.

Summala, H., Nieminen, T., \& Punto, M. (1996). Maintaining lane position with peripheral vision during in-vehicle tasks. Human Factors, 38(3), 442-451.

Taves, D. R. (1974). Minimization: A new method of assigning patients to treatment and control groups. Clinical Pharmacology and Therapeutics, 15, 443-453.

Underwood, G., Chapman, P., Bowden, K., \& Crundall, D. (2002). Visual search while driving: skill and awareness during inspection of the scene. Transportation Research Part F: Traffic Psychology and Behaviour, 5(2), 87-97.

Underwood, G., Chapman, P., Brocklehurst, N., Underwood, J., \& Crundall, D. (2003). Visual attention while driving: sequences of eye fixations made by experienced and novice drivers. Ergonomics, 46(6), 629-646. 